\title{
Evaluation of a Differential Mobility Spectrometer/Miniature Mass Spectrometer System
}

\author{
Fatkhulla K. Tadjimukhamedov, ${ }^{a}$ Ayanna U. Jackson, ${ }^{a}$ \\ Erkinjon G. Nazarov, ${ }^{b}$ Zheng Ouyang, ${ }^{c}$ and R. Graham Cooks ${ }^{a}$ \\ ${ }^{a}$ Department of Chemistry, Purdue University, West Lafayette, Indiana, USA \\ ${ }^{\mathrm{b}}$ Sionex Corporation, Bedford, Massachusetts, USA \\ ${ }^{\mathrm{c}}$ Weldon School of Biomedical Engineering, Purdue University, West Lafayette, Indiana, USA
}

A planar differential mobility spectrometer (DMS) was coupled to a Mini 10 handheld rectilinear ion trap (RIT) mass spectrometer (MS) (total weight $10 \mathrm{~kg}$ ), and the performance of the instrument was evaluated using illicit drug analysis. Coupling of DMS (which requires a continuous flow of drift gas) with a miniature MS (which operates best using sample introduction via a discontinuous atmospheric pressure interface, DAPI), was achieved with auxiliary pumping using a $5 \mathrm{~L} / \mathrm{min}$ miniature diaphragm sample pump placed between the two devices. On-line ion mobility filtering showed to be advantageous in reducing the background chemical noise in the analysis of the psychotropic drug diazepam in urine using nanoelectrospray ionization. The combination of a miniature mass spectrometer with simple and rapid gas-phase ion separation by DMS allowed the characteristic fragmentation pattern of diazepam to be distinguished in a simple urine extract at lower limits of detection $(50 \mathrm{ng} / \mathrm{mL})$ than that achieved without DMS $(200 \mathrm{ng} / \mathrm{mL})$. The additional separation power of DMS facilitated the identification of two drugs of similar molecular weight, morphine (average MW $=285.34$ ) and diazepam (average MW $=284.70$ ), using a miniature mass spectrometer capable of unit resolution. The similarity in the proton affinities of these two compounds resulted in some cross-interference in the MS data due to facile ionization of the neutral form of the compound even when the ionic form had been separated by DMS. (J Am Soc Mass Spectrom 2010, 21, 1477-1481) (C) 2010 Published by Elsevier Inc. on behalf of American Society for Mass Spectrometry

$\mathrm{R}$ ecent advances in the development of miniaturized mass spectrometers (MS) have led to the realization of handheld mass spectrometers (MS) as light as $4 \mathrm{~kg}$ with encouraging performance characteristics [1-4]. The advantages of using ion trap mass analyzers for miniature mass spectrometers lie chiefly in the intrinsic capabilities for tandem MS analysis, as well as in the relatively relaxed vacuum requirements of this type of mass analyzer. The discontinuous atmospheric pressure interface (DAPI) $[5,6]$ allows the transfer of ions, generated at atmospheric pressure, into the miniature mass spectrometer and, hence, direct analysis of complex mixtures can be achieved with a small instrument using modern atmospheric pressure ionization methods. The use of miniature mass spectrometers for ambient analysis of untreated samples is difficult, because interferences cannot be ameliorated in the usual way, i.e., by chromatographic separation to purify the sample presented to the mass spectrometer, without compromising the instrument portability or the speed of analysis. This paper demonstrates improve-

Address reprint requests to Dr. R. G. Cooks, Department of Chemistry, Purdue University, 560 Oval Drive, West Lafayette, IN 47907-1393, USA. E-mail: cooks@purdue.edu ment in the analysis of complex mixtures using a handheld mass spectrometer equipped with differential ion mobility.

Differential mobility spectrometry (DMS) [7], also known as high-field asymmetric waveform ion mobility spectrometry (FAIMS) [8,9], is a form of ion mobility spectrometry (IMS) [10] that separates gas-phase ions at ambient pressure. Unlike drift-time IMS instruments, where ions are separated based on their intrinsic mobilities $(\mathrm{K})$ in relatively low electric fields $(\mathrm{E}<500$ $\mathrm{V} / \mathrm{cm}$ at ambient pressure), separations with DMS are based on differences of ion mobilities $\left(\Delta \mathrm{K}=\mathrm{K}_{\mathrm{h}}-\mathrm{K}_{\mathrm{l}}\right)$ in the high $\left(\mathrm{E}_{\mathrm{h}} \sim\right.$ up to $\left.30,000 \mathrm{~V} / \mathrm{cm}\right)$ and low $\left(\mathrm{E}_{1}\right)$ electric field regimes. Ions are carried through the DMS using a gas stream and filtered out based on differences in path due to uncompensated high and low field dispersion. Analyzers with micromachined DMS drift tubes have been used previously for monitoring volatile organic compounds, chemical warfare agents, and explosives $[11,12]$. However, it is difficult to achieve unequivocal detection and characterization of these compounds with DMS alone if they are present in complex mixtures. (Similar considerations apply to IMS separations.) Driven by this consideration, DMS systems have been successfully demonstrated as ion pre-filters on bench- 
top MS instruments $[8,13]$ displaying the advantages of reducing background noise as well as separating isomeric and isobaric species. This capability is especially attractive for miniature mass spectrometers given that only moderate mass resolution is available.

In this work, a small planar DMS drift tube [14] was coupled to a portable Mini 10 mass spectrometer [15] with a DAPI interface, and the advantages of this hybrid configuration were tested in the analysis of an illicit drug in a complex matrix. An auxiliary air flow between the DMS and DAPI was established to provide stable gas flow needed for ion transfer through the DMS drift tube.

\section{Experimental}

The DMS unit used in these measurements was model SVACG (Sionex Corp., Bedford, MA, USA); the drift tube was of a planar configuration with the following dimensions: $10 \mathrm{~mm}$ long, $4 \mathrm{~mm}$ wide with a separation gap of $0.5 \mathrm{~mm}$ (Figure 1). The frequency of the waveform was set at $1.2 \mathrm{MHz}$ and experiments were carried out at rf high-field amplitudes $\left(\mathrm{V}_{\mathrm{h}}\right)$ of 1250 or $1500 \mathrm{~V}$, which correspond to separation fields $(\mathrm{E} / \mathrm{N}$, in units of Townsend, Td) of 105 or $127 \mathrm{Td}(1 \mathrm{Td} \approx 240 \mathrm{~V} / \mathrm{cm}$ at ambient pressure and temperature). The low field amplitudes used were 450 or $543 \mathrm{~V}$, respectively. The resolving power of the DMS instrument was similar to the values of 20-30 reported earlier [13]. Standard Mini 10 operating parameters were used for the measurements (see Supplementary Information, which can be found in the electronic version of this article).

Standard $1 \mathrm{mg} / \mathrm{mL}$ solutions of morphine and diazepam were obtained from Cerilliant Corp. (Round Rock, TX, USA) and were further diluted in methanol with
$0.1 \%$ formic acid (FA). Ionization was made by nanoESI in the positive ion mode. A healthy human urine sample, spiked with diazepam at selected concentrations, was used with a simple separation step to isolate diazepam from the matrix. (See Supplementary Material for details.) The nano-ESI capillaries were prepared with model P-97 Micropipette Puller (Sutter Instruments, Novato, CA, USA). The nano-ESI source was operated at $1.5 \mathrm{kV}$ and the spray flow rate was 200 $\mathrm{nL} /$ min measured at this voltage.

\section{Results and Discussion}

Experiments were carried out with the DMS drift tube attached to the Mini 10 instrument, equipped with a DAPI unit, Figure 1. The flow rate of the gas admitted into the vacuum manifold with DAPI during the open period was measured at $\sim 0.0012 \mathrm{~L} / \mathrm{min}$. For optimum DMS performance, a minimum of $0.3 \mathrm{~L} / \mathrm{min}$ continuous gas flow is required. Thus, to effectively couple the DMS and mini MS, an interface with an auxiliary suction was employed between the DMS filter and the DAPI. This allows independent operation of both the DMS operating in the continuous filter mode and the Mini 10 operating with pulsed sample introduction. The interface was constructed from a $5 \mathrm{~cm}$ long, $1 / 4$ in. o.d. stainless steel tube and 1/4 in. Swagelok tee. A small diaphragm pump ( $5 \mathrm{~L} / \mathrm{min}$ ) was attached to the tee and the gas flow (laboratory air) was restricted to $\sim 0.8-1$ $\mathrm{L} / \mathrm{min}$ using a rotameter (Key Instruments, Trevose, PA, USA). The resulting linear velocity of the drift gas (lab air) through the DMS channel is estimated at $\sim 6$ $\mathrm{m} / \mathrm{s}$. The Mini 10 mass spectrometer uses a rectilinear ion trap as the mass analyzer and unit mass resolution is achieved for analysis. Although isomeric or isobaric

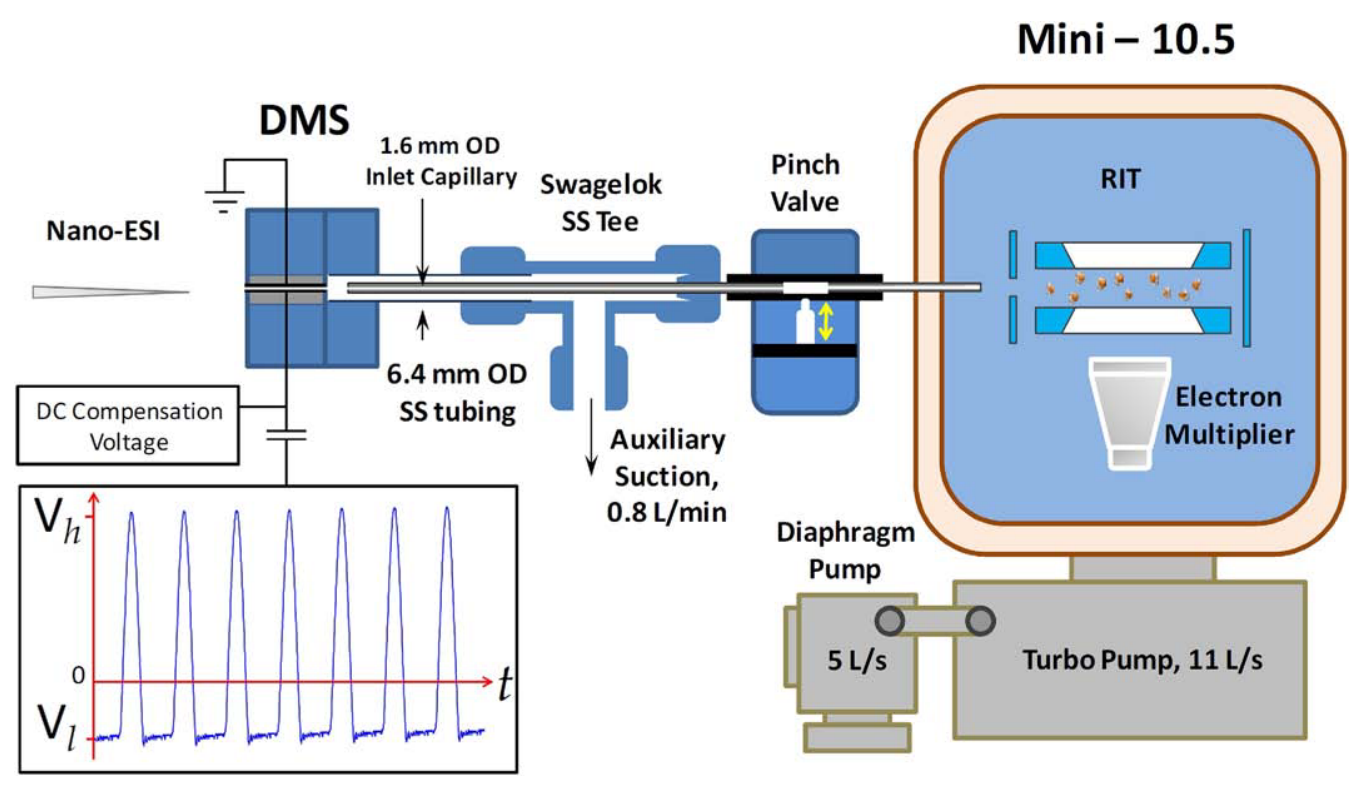

Figure 1. Schematic of the instrument configuration with a nano-ESI, a DMS and a Mini 10 handheld mass spectrometer equipped with a rectilinear ion trap (RIT) mass analyzer. The instrumentation is not drawn to scale. 
compounds can be differentiated using tandem MS analysis, the efficiency of trapping and isolating analyte ions can be significantly impacted by the total number of the ions generated from the sample and by the relative concentrations of the mixture components [16].

The Mini 10 mass spectra, shown in Figure 2, represent experimental data obtained without (left column) and with DMS pre-filtering (right column). One of the advantages of planar DMS is that it offers two modes of operation: (1) transparent mode, which transmits all ions without discrimination or major ion loss, and (2) ion-filter mode, which allows differential mobility selection of ions. Switching between modes is achieved simply by turning the separation field on and off [17]. The mass spectra shown in Figure 2a, c, and e were recorded in the transparent DMS mode (i.e., with the DMS drift tube in place but the electrodes grounded) for an SPE extract of a urine sample containing diazepam at $200 \mathrm{ppb}$ ionized with nano-ESI. The zoomed-in window, shown in the inset of Figure 2a, displays a broad distribution of overlapping peaks in the mass spectrum between $\mathrm{m} / \mathrm{z} 280$ and 290 , covering the region of protonated diazepam at $\mathrm{m} / \mathrm{z} 285$. The spectrum also displays urine metabolite peaks with substantial intensities at $m / z$ 303, 310, 358, and many other ions. Using tandem MS analysis, the ion at $m / z 114$ was confirmed as protonated creatinine (MW 113.12), a common urine metabolite that forms a fragment ion at $\mathrm{m} / \mathrm{z} 86$. Simi- larly, $m / z 174$ was identified as an adduct ion of protonated creatinine with urea.

When the DMS filter was activated and operated at $\mathrm{V}_{\mathrm{h}}=1500 \mathrm{~V}$ (Figure $2 \mathrm{~b}$ ), a DMS compensation voltage $(\mathrm{CV})$ of $\sim 6.0 \mathrm{~V}$ filters out most other ions and leaves the diazepam ions $(m / z 285)$. The peak intensity at $m / z 285$ in this spectrum is almost 1300 units-a significant increase when compared to the spectrum shown in Figure 2a. This is attributed to reduced levels of space charge effects and/or ion suppression (by matrix ions in the course of ion/molecule reactions) as a result of filtering with DMS. The isolation of the precursor ions without and with differential mobility selection is shown in Figure $2 c$ and $d$, respectively. The spectrum shows much cleaner isolation and higher signal-tonoise ratios $(\mathrm{S} / \mathrm{N})$ when isolation was obtained after the DMS filtering (with improvements in $\mathrm{S} / \mathrm{N}$ up to nine). The amplitude of the notched waveform during ion isolation in these spectra was set at $5.0 \mathrm{~V}$ and the intensity of the $m / z 285$ peak decreased from 1300 to 240 units (Figure $2 b$ and $d$ ) as a result of isolation. Optimization of $m / z 285$ ion isolation, without DMS filtering, was tried using a variety of different conditions, including varying the amplitude of the notched waveform and the width of the isolation widow. Improvements in isolation could be achieved, but only at the cost of significant loss in the intensity of the ion $m / z 285$, most likely the result of the loss of resolution due to space (a)

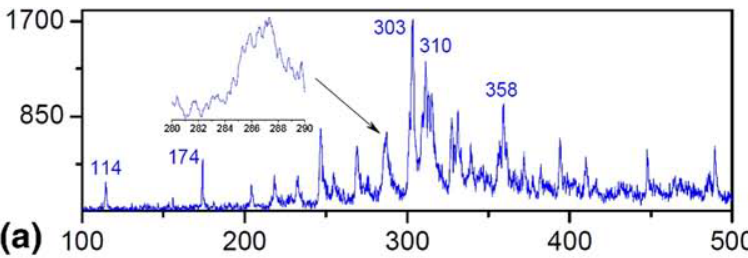

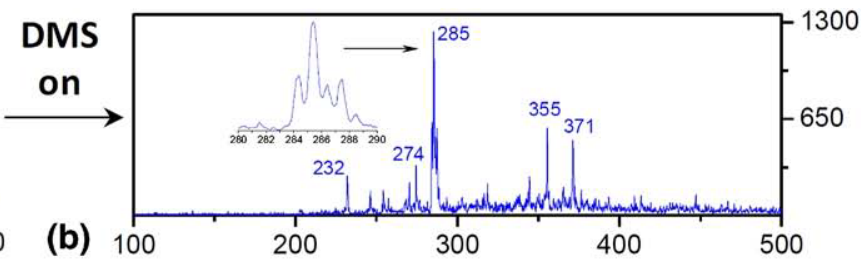

(b) 100

Ion Isolation, $\mathrm{m} / \mathrm{z} 285$

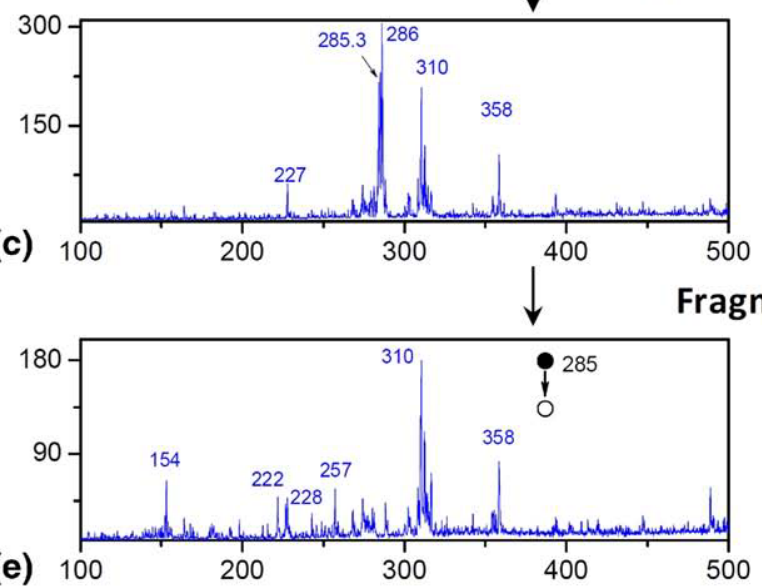

(d)

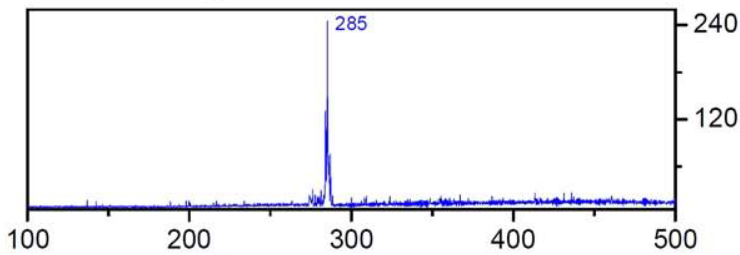

(f)

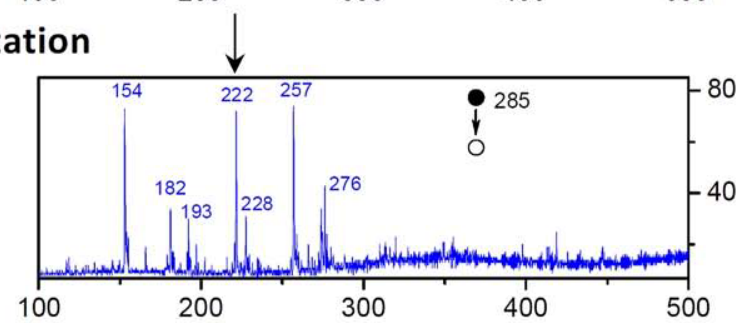

$\mathrm{m} / \mathrm{z}$

Figure 2. Comparison of analysis of an extract of $200 \mathrm{ppb}$ diazepam in urine using Mini 10 without (left column) and with (right column) DMS separation: (a), (b) full mass spectra; (c), (d) isolation of $\mathrm{m} / \mathrm{z}$ 285; and (e), (f) fragmentation of isolated $\mathrm{m} / \mathrm{z} 285$ ion. DMS separation conditions: $\mathrm{V}_{\mathrm{h}}=1500 \mathrm{~V}$ and $\mathrm{CV}=-6.0 \mathrm{~V}$. 
charge effects in the ion trap. Attempts at ion isolation similar to that shown in Figure 2d, but without DMS filtering, resulted in a decrease by a factor of six or more in the intensity of the isolated peak. The corresponding MS/MS mass spectra (plots in Figure 2e and $\mathrm{f}$ ) show the main fragment ions of diazepam $(\mathrm{m} / \mathrm{z} 257,228,222$, and 154; note the presence of product ions due to ion/ molecule reactions at $m / z$ values above that of the precursor ion). The ion at $m / z 276$ (Figure $2 \mathrm{f}$ ) is not a fragment of diazepam and is likely due to imperfect ion isolation or ion/molecule reactions occurring in the ion trap. This demonstrates the key benefit of filtering ions with DMS before mass analysis and MS/MS interrogation: higher quality tandem mass spectra with characteristic fragmentation patterns could be obtained at lower analyte concentrations when pre-filtered with DMS (Supplementary Material).

Another advantage of coupling the DMS to the Mini MS is the differentiation of nearly isobaric compounds, as shown in Figure 3. In this case, a mixture of diazepam and morphine in methanol $(0.1 \% \mathrm{FA})$ was analyzed using nano-ESI and the DMS-Mini 10 mass spectrometer. The drugs differ in average molecular weight by only $0.60 \mathrm{Da}$, but have different molecular structures and elemental compositions. With the DMS turned off, the mixture yielded a mass spectrum with poorly separated peaks of protonated diazepam and morphine
(Figure 3a). The MS/MS spectrum obtained for ions at $\mathrm{m} / \mathrm{z} 286$ without DMS separation shows a set of fragment ions from both compounds (Figure 3b). With DMS filtering optimized, the best selection occurs at $\mathrm{CV}=$ $-5.0 \mathrm{~V}$ for diazepam (Figure $3 \mathrm{c}$ ) and $\mathrm{CV}=-7.5 \mathrm{~V}$ for morphine (Figure $3 \mathrm{~d}$ ) with the dispersion voltage $V_{h}$ set at $1250 \mathrm{~V}$. The insets in these figures, covering the $\mathrm{m} / \mathrm{z}$ range between $m / z 284$ and 290, show that the protonated forms of both drugs are well separated and can be independently monitored. The diazepam molecule has a single chlorine atom thus displaying a ${ }^{37} \mathrm{Cl}$ peak at $\mathrm{m} / \mathrm{z}$ 287 (Figure 3c), while a molecular ion at $m / z 286$ was observed for morphine. Fragment ion patters obtained from MS/MS spectra with DMS filtering confirm the identities of diazepam and morphine (Figure $3 e$ and f), although some interference remains with DMS separation, as shown with fragment ions marked in the spectra. The interference can be due to the ions generated from ion/molecule proton exchange reactions inside and after the DMS drift tube. This occurs when both compounds have similar proton affinities (PA) (see Supplementary Material).

\section{Conclusions}

Gas-phase ion separation with DMS followed by mass analysis using a miniature mass spectrometer is dem-

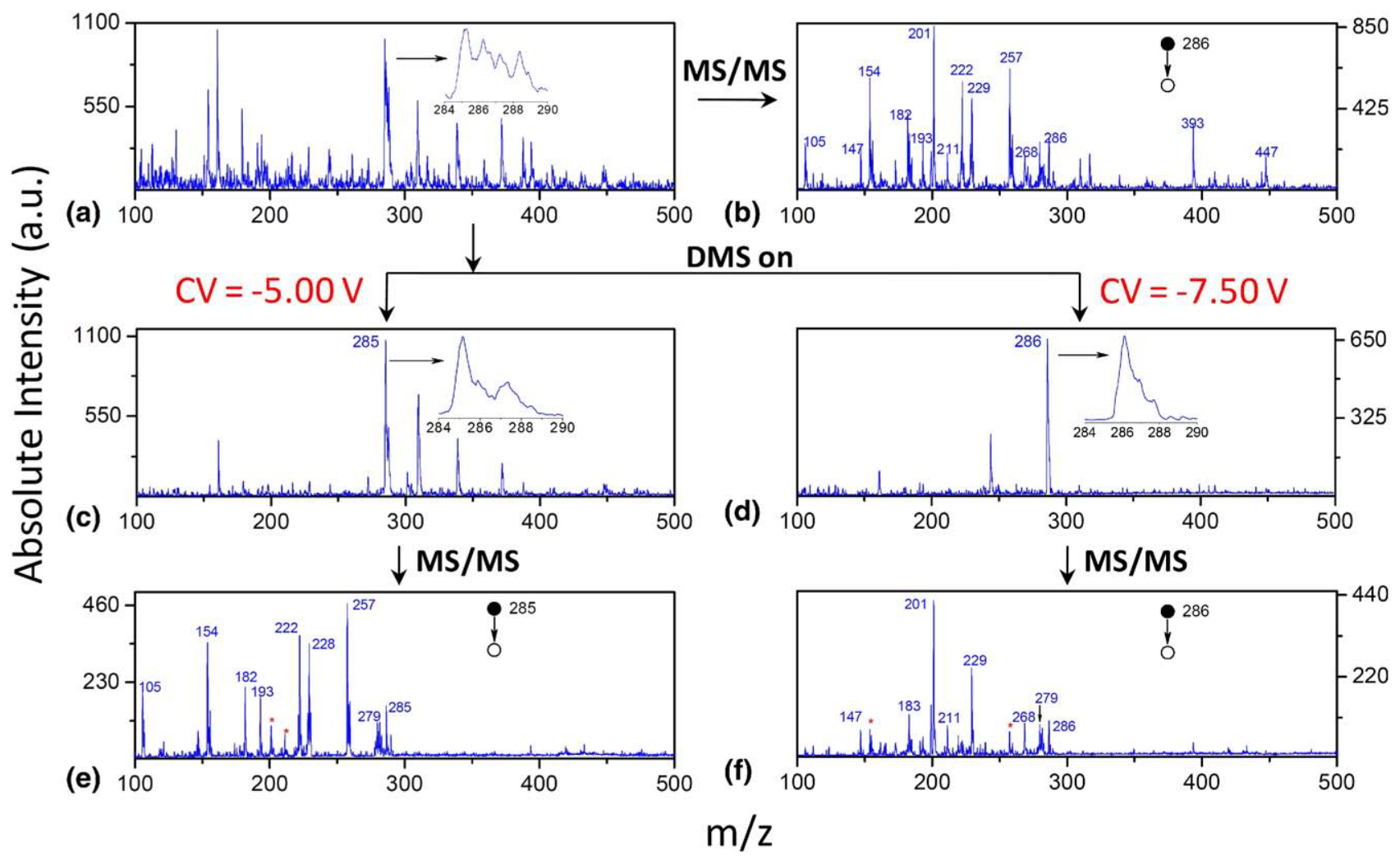

Figure 3. Nano-ESI mass spectra of a methanol solution $(0.1 \%$ FA) with $200 \mathrm{ppb}$ morphine and diazepam in the mixture. MS (a) and MS/MS (b) mass spectra of the mixture recorded without DMS filtering, MS and MS/MS mass spectra recorded with DMS separation at CV $=-5.0 \mathrm{~V}$ for diazepam (c) and (e) and at $-7.5 \mathrm{~V}$ for morphine $(\mathbf{d})$ and (f). $\mathrm{V}_{\mathrm{h}}$ was set at $1250 \mathrm{~V}$. 
onstrated to be useful in the analysis of complex samples. While a DMS analyzer has a limited resolving power in comparison with MS, the combination of the two techniques enhances the analytical power of the miniature MS system; it increases its specificity and sensitivity in the analysis of complex mixtures due to decreases in chemical noise in the mass spectra. Improvements in the S/N values for both MS and MS/MS spectra and reduced suppression of analyte signals by background ions were achieved with DMS filtering, as shown in the example of analyzing diazepam in urine samples. Separation of morphine and diazepam in DMS showed that the differentiation of isobaric compounds can be improved with differential mobility separations.

\section{Acknowledgments}

The authors acknowledge support for this work by DTRA under contract no. N00164-08-C-JQ11. The assistance of Anthony Costa with electronic structure calculations is also acknowledged.

\section{Appendix A \\ Supplementary Material}

Supplementary material associated with this article may be found in the online version at doi:10.1016/ j.jasms.2010.06.001.

\section{References}

1. Ouyang, Z.; Cooks, R. G. Miniature Mass Spectrometers. Annu. Rev. Anal. Chem. 2009, 2, 187-214.

2. March, R. E.; Todd, J. F. J. Quadrupole Ion Trap Mass Spectrometry, 2nd ed.; John Wiley and Sons, Inc.: Hoboken, NJ, 2005; pp 175-179, 189-210.
3. Whitten, W. B.; Reilly, P. T. A.; Ramsey, J. M. High-Pressure Ion Trap Mass Spectrometry. Rapid Commun. Mass Spectrom. 2004, 18, 1749-1752.

4. Griest, W. H.; Lammert, S. A. In Chemical Analysis, Vol. CLXIX; Winefordner, J. D., Ed.; Wiley and Sons, Inc.: Hoboken, NJ, 2006; pp 61-89.

5. Gao, L.; Cooks, R. G.; Ouyang, Z. Breaking the Pumping Speed Barrier in Mass Spectrometry: Discontinuous Atmospheric Pressure Interface. Anal. Chem. 2008, 80, 4026-4032.

6. Gao, L.; Li, G. T.; Nie, Z. X.; Duncan, J.; Ouyang, Z.; Cooks, R. G. Characterization of a Discontinuous Atmospheric Pressure Interface. Multiple Ion Introduction Pulses for Improved Performance. Int. J. Mass Spectrom. 2009, 283, 30-34.

7. Buryakov, I. A.; Krylov, E. V.; Nazarov, E. G.; Rasulev, U. K. A New Method of Separation of Multi-Atomic Ions by Mobility at Atmospheric Pressure Using a High-Frequency Amplitude-Asymmetric Strong Electric Field. Int. J. Mass Spectrom. Ion Processes 1993, 128, 143-148.

8. Guevremont, R.; Purves, R. W. High Field Asymmetric Waveform Ion Mobility Spectrometry-Mass Spectrometry: An Investigation of Leucine Enkephalin Ions Produced by Electrospray Ionization. J. Am. Soc. Mass Spectrom. 1999, 10, 492-501.

9. Shvartsburg, A. A. Differential Ion Mobility Spectrometry: Nonlinear Ion Transport and Fundamentals of FAIMS; CRC Press: Boca Raton, 2009.

10. Eiceman, G. A.; Karpas, Z. Ion Mobility Spectrometry, 2nd ed; Taylor and Francis Group: Boca Raton, FL, 2005.

11. Krebs, M. D.; Zapata, A. M.; Nazarov, E. G.; Miller, R. A.; Costa, I. S. Sonenshein, A. L.; Davis, C. E. Detection of Biological and Chemical Agents Using Differential Mobility Spectrometry Technology. IEEE Sens. J. 2005, 5, 696-703.

12. Eiceman, G. A.; Krylov, E. V.; Krylova, N. S.; Nazarov, E. G.; Miller, R. A. Separation of Ions from Explosives in Differential Mobility Spectrometry by Vapor-Modified Drift Gas. Anal. Chem. 2004, 76, 4937-4944.

13. Levin, D. S.; Vouros, P.; Miller, R. A.; Nazarov, E. G. Using a Nanoelectrospray-Differential Mobility Spectrometer-Mass Spectrometer System for the Analysis of Oligosaccharides with Solvent Selected Control Over ESI Aggregate Ion Formation. J. Am. Soc. Mass Spectrom. 2007, 18, 502-511.

14. Miller, R. A.; Nazarov, E.; Coy, S. L.; Krylov, E. Miniature Differential Mobility Spectrometer as a Pre-Filter for Atmospheric-Pressure Mass Spectrometry. Int. J. Ion Mobility Spectrom. 2006, 9, 35-39.

15. Gao, L.; Song, Q.; Patterson, G. E.; Cooks, R. G.; Ouyang, Z. Handheld Rectilinear Ion Trap Mass Spectrometer. Anal. Chem. 2006, 78, 59946002.

16. Schwartz, J. C.; Senko, M. W.; Syka, J. E. P. A Two-Dimensional Quadrupole Ion Trap Mass Spectrometer. J. Am. Soc. Mass Spectrom. 2002, 13, 659-669.

17. Schneider, B. B.; Covey, T. R.; Coy, S. L.; Krylov, E. V.; Nazarov, E. G. Planar Differential Mobility Spectrometer as a Pre-Filter for Atmospheric Pressure Ionization Mass Spectrometry. Int. J. Mass Spectrom. in press. 